\title{
Analysis of Heat and Mass Transfer in a Tapered Asymmetric Channel During Peristaltic Transport of (Pseudoplastic Nanofluid) with Variable Viscosity Under the Effect of (MHD)
}

\author{
Mohammed R. Salman Ahmed M. Abdulhadi \\ University of Baghdad-College of Science-Department of Mathematics \\ mawb1967@gmail.com \\ ahm6161@yahoo.com
}

Available online : $\quad 6 / 8 / 2018$

DOI: $10.29304 / j q c m .2018 .10 .3 .408$

\begin{abstract}
.
In this paper, a study and an analysis of a heat and mass transfer during peristaltic flow for a pseudoplastic fluid in asymmetric tapered channel, and a variable viscosity dependent of a fluid temperature with exist of slip conditions through porous medium and the influence of this conditions on the velocity and pressure, where the wavelength of the peristaltic flow is a long and the Reynold number is very small. The solution of equations for the momentum and energy have been on the basis of a perturbation technique for a found the stream function, velocity, pressure gradient and temperature and also have been discussed the trapping phenomenon by the graphs. Key words. Peristaltic transport,Reynolds number, Hartmann number, pseudoplastic, Porous Medium
\end{abstract}

Mathematics subject classification: $80 \mathrm{Axx}$, 76Dxx, 76Txx. 


\section{Introduction:}

The process of peristalsis has become a subject of great importance for the researchers in view of its wide-ranging biomedical, physiological applications. You can see it in the food movement in the Intestine tract,the urine passage from a kidney to the bladder, Blood transfusion in the capillary blood vessels, also machines used in heart and lung operations and the movement of sperm in the male reproductive system and the transfer of the egg in the womb in women. Latham [1] and the physiology of the gastrointestinal tract, esophagus, stomach, intestine, and ureter associated with the phenomenon of peristalsis was discussed in the book "Biomathematics"[2].Also on the industrial field where has encouraged the complex nature of fluids of which, oils, chemicals, petroleum and other fluids, has encouraged extensive studies and research into the properties of these fluids. where many researchers presented basic equations for various non-Newtonian liquids [3-7].

Heat transfer in peristalsis is beneficial in the applications such as blood pumps in heart operations, Kidney dialysis operations, and Magnetohydrodynamics (MHD) is a topic important to many researchers in the problems they treated conductive fluids e.g., blood, blood pumping appliances, magnetic resonance imaging (MRI) for brain diagnosis. MHD has many implementations in geology (in the study of earthquakes and the subsoil of the earth) [8-15].

The word nanofluid is referring to a fluid containing nanometer-sized particles. Choi [16], the Nanofluids have applications in numerous medical, biochemical and engineeering including neuroelectronic interfaces, nanoporous materials (carbon, nanofibers), cancer diagnosis, drug delivery systems and many others.

The pseudoplastic fluids consider is a category of shear fluffy materials. In this materials, the viscosity decreases by enhancing shear rate. it is clear that non-Newtonian materials are involved in many qualities and ingredients and processes including food mixing, food movement in the intestine, blood flow in arteries and capillaries, the flow of metal fluids and alloys.
Most of the researchers on the peristalsis channels studies consider fluid viscosity is constant ,But some of them showed great importance to the situations which can attention variable viscosity of the fluid.And from these [17-24].

In a study recent also, Misra et al.[25] the influence of heat and mass transfer in asymmetric channels during peristaltic transport of an MHD fluid having temperature-dependent properties and Sinha et al.[26] Peristaltic transport of MHD flow and heat transfer in an asymmetric channel: effects of variable viscosity, velocity-slip and temperature jump and Hayat et al. [27] Influence of convective conditions in radiative peristaltic flow of pseudoplastic nanofluid in a tapered asymmetric channel.

In this paper, we will study the heat and mass transfer in a tapered asymmetric channel under the effect of a magnetohydrodynamic during peristaltic transport of pseudoplastic nanofluid with slip conditions in peristaltic flow for a variables viscosity for this fluid, where the wavelength of the peristaltic flow is long and the Reynolds number is small. The equations for the momentum and energy have been linearized on the basis of these considerations. Expressions for the stream function, velocity, pressure gradient and temperature have been obtained. Pumping characteristics of the peristaltic flow and the trapping phenomenon have been discussed, and we obtained numerical results of different physical parameters and a graphs by using the software MATHEMATICA. Accordingly, we will analyzed these data based on these figures.

\section{Mathematical Formulation:}

In the present study, we consider the flow of an incompressible magnetohydrodynamic (MHD) pseudoplastic nanofluid in a two-dimensional tapered asymmetric channel through a porous medium (see Fig.(1)) and the flux is induced by sinusoidal wave traveling propagating with constant velocity c along the channel walls and the effect this on a heat and mass transfer with a velocity of peristaltic waves. its walls are defined as:

$$
\begin{aligned}
& \tilde{Y}=\tilde{H}_{1}\left(\tilde{X}, t^{\prime}\right)=-d_{1}-m^{\prime} \tilde{X} \\
&-b_{1} \operatorname{Sin}\left[\frac{2 \pi}{\lambda}\left(\tilde{X}-c t^{\prime}\right)+\phi\right]
\end{aligned}
$$


$\tilde{Y}=\tilde{H}_{2}\left(\tilde{X}, t^{\prime}\right)=d_{2}+m^{\prime} \tilde{X}$

$$
+b_{2} \operatorname{Sin}\left[\frac{2 \pi}{\lambda}\left(\tilde{X}-c t^{\prime}\right)\right]
$$

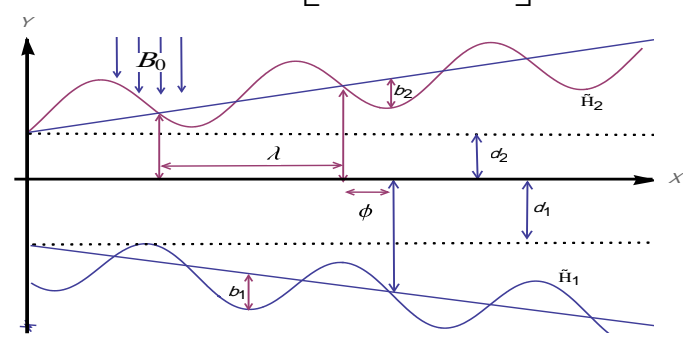

Fig.(1): Geometry of the tapered asymmetric channel.

Where $\left(d_{1}\right),\left(d_{2}\right)$ is the channel width, $\left(\mathrm{b}_{1}\right)$ and $\left(b_{2}\right)$ are the amplitudes of right and left walls respectively, (c) is the phase speed of the wave, $m^{\prime}(\square 1)$ is the non-uniform parameter, $(\lambda)$ is the wave length, $\left(t^{\prime}\right)$ the time, the phase difference $(\phi)$ varies in the range $(0 \leq \phi \leq \pi)$ where $(\phi=0)$ corresponds to symmetric channel, with waves out of phase i.e. both walls move towards outward or inward simultaneously and further $b_{1}, b_{2}, d_{1}, d_{2}, \phi$ satisfy the following condition at the inlet of a divergent channel.

$b_{1}^{2}+b_{2}^{2}+2 b_{1} b_{2} \operatorname{Cos} \phi \leq\left(d_{1}+d_{2}\right)^{2}$

Here we assume the fluid to be electrically conducting in the presence of a magnetic field $B=\left(0, B_{0}, 0\right)$. To calculate the Lorentz force we will apply a magnetic field just in $\tilde{Y}-$ direction and then we study the effect of it on the fluid flow.

$$
\begin{aligned}
& \tilde{S}_{\tilde{X} \tilde{X}}+\lambda_{1}\left[\left(\frac{\partial}{\partial t^{\prime}}+\tilde{U} \frac{\partial}{\partial \tilde{X}}+\tilde{V} \frac{\partial}{\partial \tilde{Y}}\right) \tilde{S}_{\tilde{X} \tilde{X}}-2 \tilde{S_{X \tilde{X}}} \frac{\partial \tilde{U}}{\partial \tilde{X}}-2 \tilde{S_{X} \tilde{Y}} \frac{\partial \tilde{U}}{\partial Y^{\tilde{r}}}\right] \\
& +\frac{1}{2}\left(\lambda_{1}-\mu_{1}\right)\left[4 \tilde{S}_{\tilde{X} \tilde{X}} \frac{\partial \tilde{U}}{\partial \tilde{X}}+2 \tilde{S_{\tilde{Y}}}\left(\frac{\partial \tilde{U}}{\partial Y^{\tau}}+\frac{\partial \tilde{V}}{\partial \tilde{X}}\right)\right]=2 \mu_{0} \frac{\partial \tilde{U}}{\partial \tilde{X}} \\
& \tilde{S}_{\tilde{X Y}}{ }+\lambda_{1}\left[\left(\frac{\partial}{\partial t^{\prime}}+\tilde{U} \frac{\partial}{\partial \tilde{X}}+\tilde{V^{2}} \frac{\partial}{\partial Y^{\sim}}\right) \tilde{S}_{\tilde{X Y} Y^{\sim}}-\tilde{S_{X \tilde{X}}} \frac{\partial \tilde{V^{\prime}}}{\partial \tilde{X}}-\tilde{S_{Y_{Y}}} \frac{\partial \tilde{U}}{\partial Y^{\sim}}\right] \\
& +\frac{1}{2}\left(\lambda_{1}-\mu_{1}\right)\left(\tilde{S_{\tilde{X} \tilde{X}}}+\tilde{S}_{\tilde{Y} \tilde{Y}}\right)\left(\frac{\partial \tilde{U}}{\partial \tilde{Y}}+\frac{\partial \tilde{V}}{\partial \tilde{X}}\right)=\mu_{0}\left(\frac{\partial \tilde{U}}{\partial \tilde{Y}}+\frac{\partial \tilde{V}}{\partial \tilde{X}}\right)
\end{aligned}
$$

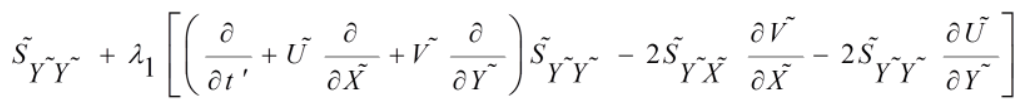

$$
\begin{aligned}
& +\frac{1}{2}\left(\lambda_{1}-\mu_{1}\right)\left[2 \tilde{S_{X Y}}\left(\frac{\partial \tilde{U}}{\partial \tilde{Y}}+\frac{\partial \tilde{V}}{\partial \tilde{X}}\right)+4 \tilde{Y_{Y \sim Y^{\tau}}} \frac{\partial \tilde{V}}{\partial \tilde{Y}}\right]=2 \mu_{0} \frac{\partial \tilde{V^{\tau}}}{\partial \tilde{Y}}
\end{aligned}
$$




\section{Governing Equations:}

Based on the consideration made above, the governing equations that describe the flow in the present study as follows:

$$
\begin{aligned}
& \frac{\partial \tilde{U}}{\partial \tilde{X}}+\frac{\partial \tilde{V}}{\partial \tilde{Y}}=0 \\
& \rho_{f}\left(\frac{\partial \tilde{U}}{\partial t^{\prime}}+\tilde{U} \frac{\partial \tilde{U}}{\partial \tilde{X}}+\tilde{V} \frac{\partial \tilde{U}}{\partial \tilde{Y}}\right)=-\frac{\partial \tilde{P}}{\partial \tilde{X}}+\frac{\partial}{\partial \tilde{X}}(\tilde{S} \tilde{X} \tilde{X})+\frac{\partial}{\partial \tilde{Y}}(\tilde{S} \tilde{X} \tilde{Y})-\sigma^{\prime} B_{0}^{2} \tilde{U}-\frac{\tilde{\mu}(\tilde{T})}{K_{0} \tilde{U}} \\
& \rho_{f}\left(\frac{\partial \tilde{V}}{\partial t^{\prime}}+\tilde{U} \frac{\partial \tilde{V}}{\partial \tilde{X}}+\tilde{V} \frac{\partial \tilde{V}}{\partial \tilde{Y}}\right)=-\frac{\partial \tilde{P}}{\partial \tilde{Y}}+\frac{\partial}{\partial \tilde{Y}}\left(\tilde{S_{\tilde{Y} \tilde{Y}}}\right)+\frac{\partial}{\partial \tilde{X}}\left(\tilde{S}_{\tilde{Y} \tilde{X}}\right)-\frac{\tilde{\mu}(\tilde{T})}{K_{0}} \\
& \left(\rho c^{\prime}\right)_{f}\left[\frac{\partial \tilde{T}}{\partial t^{\prime}}+\tilde{U} \frac{\partial \tilde{T}}{\partial \tilde{X}}+\tilde{V} \frac{\partial \tilde{T}}{\partial \tilde{Y}}\right]=\kappa\left[\frac{\partial^{2} \tilde{T}}{\partial \tilde{X}^{2}}+\frac{\partial^{2} \tilde{T}}{\partial \tilde{Y}^{2}}\right]+\left(\rho c^{\prime}\right)_{p} \frac{D_{T}}{T_{m}}\left[\left(\frac{\partial \tilde{T}}{\partial \tilde{X}}\right)^{2}+\left(\frac{\partial \tilde{T}}{\partial \tilde{Y}}\right)^{2}\right]
\end{aligned}
$$

In above equations, $\left(t^{\prime}\right)$ denotes the dimensional time, $(\tilde{P})$ the pressure, $\left(\tilde{S_{\tilde{X} \tilde{X}}}, \tilde{S_{\tilde{X} \tilde{Y}}},{\tilde{S_{Y \tilde{X}}}}, \tilde{S_{\tilde{Y Y}}}\right)$ the components of stress tensor, $\left(\sigma^{\prime}\right)$ the fluid electrical conductivity,

$(\tilde{U})$ and $(\tilde{V})$ are the velocity components in the axial and transverse directions respectively,

$(\kappa)$ the thermal conductivity of fluid, $\left(B_{0}\right)$ is the magnetic parameter, $\left(\rho_{f}\right)$ the density of fluid, $\left(\rho_{p}\right)$ the density of nano-particles, $\tilde{\mu}(\tilde{T})$ the variable viscosity, $(\tilde{T})$ the temperature of fluid, $\left(T_{m}\right)$ the fluid mean temperature, $\left(D_{T}\right)$ the thermophoretic diffusion coefficient, $\left(\mu_{0}\right)$ a constant viscosity and $\left(K_{0}\right)$ the permeability parameter.

The appropriate boundary conditions comp-rising wall slip and convective boundary cond-itions are given as follows:

$$
\begin{aligned}
\tilde{u} & =-c, & \tilde{T}=T_{0} & \text { at } & \tilde{Y} & =\tilde{H}_{1} \\
\tilde{u} & =-c, & \tilde{T}=T_{1} & \text { at } & \tilde{Y} & =\tilde{H}_{2}
\end{aligned}
$$

Now we treat the wave frame having coordinates $(\mathrm{X}, \mathrm{Y})$ moving in the $\mathrm{X}$-direction with wave velocity (c). The velocities, pressure, time and coordinates in two frames are a related by:

$$
\left.\begin{array}{l}
\tilde{x}=\tilde{X}-c t^{\prime}, \tilde{y}=\tilde{Y^{\prime}}, \tilde{u}(\tilde{x}, \tilde{y})=\tilde{U}\left(\tilde{X}, \tilde{Y}, t^{\prime}\right)-c, \\
\tilde{v}(\tilde{x}, \tilde{y})=\tilde{V}\left(\tilde{X}, \tilde{Y}, t^{\prime}\right), \tilde{p}(\tilde{x}, \tilde{y})=\tilde{P}\left(\tilde{X}, \tilde{Y}, t^{\prime}\right)
\end{array}\right\}
$$

Where $\tilde{u}, \tilde{v}$ are the velocity components in the wave frame $(\tilde{x}, \tilde{y})$ and $\tilde{p}, \tilde{P}$ are the pressures in the wave and laboratory setting respectively.

Now we will define the non-dimensional quantities and stream function through the equations below:

$$
\begin{aligned}
& x=\frac{\tilde{x}}{\lambda}, y=\frac{\tilde{y}}{d_{1}}, t=\frac{c t^{\prime}}{\lambda}, u=\frac{\tilde{u}}{c}, v=\frac{\tilde{v}}{c}, \delta=\frac{d_{1}}{\lambda}, \theta=\frac{T-T_{0}}{T_{1}-T_{0}}, \\
& \mu(\theta)=\frac{\tilde{\mu}(T)}{\mu_{0}}, h_{1}(x)=\frac{\tilde{H}_{1}(\tilde{x})}{d_{1}}, h_{2}(x)=\frac{\tilde{H}_{2}(\tilde{x})}{d_{1}}, \\
& p=\frac{d_{1}^{2} \tilde{p}}{\lambda \mu_{0} c}, S_{i j}=\frac{d_{1}}{c \mu_{0}} \tilde{S}_{i j}, m=\frac{m^{\prime} \lambda}{d_{1}}, K=\frac{K_{0}}{d_{1}^{2}}, \\
& a=\frac{b_{1}}{d_{1}}, b=\frac{b_{2}}{d_{1}}, R_{e}=\frac{\rho_{f} c d_{1}}{\mu}, P_{r}=\frac{\mu_{0} c_{f}^{\prime}}{\kappa}, \\
& N t=\frac{\dot{\tau} D_{T}\left(T-T_{0}\right)}{T_{m} v}, M=\sqrt{\frac{\sigma^{\prime}}{\mu}} d_{1} B_{0}, \\
& u=\frac{\partial \psi}{\partial y}, v=-\delta \frac{\partial \psi}{\partial x}
\end{aligned}
$$

Where $(\psi)$ is the stream function, $(x)$ is the nondimensional axial coordinate, (y) is the nondimensional transverse coordinate, $(\mathrm{t})$ is the dimensionless time, (u) and (v) are non-dimensional axial and transverse velocity components respectively, (p) is the dimensionless pressure, (a) and (b) are amplitudes of upper and lower walls, $(\delta)$ is the wave number, (m) is the non-uniform parameter, $(\mathrm{K})$ is the Darcy number, $(\mathrm{Re})$ is the Reynolds number, $(v)$ is the nanofluid kinematic viscosity, $\dot{\tau}=\left(\rho c^{\prime}\right)_{p} /\left(\rho c^{\prime}\right)_{f}$ is the ratio of the effective heat capacity of nanoparticle 
material and heat capacity of the fluid, $(\theta)$ is the dimensionless temperature, $\left(P_{r}\right)$ is the Prandtl number, (M) is the Hartmann number, $\left(\rho_{p}\right)$ the density of nano-particles, $(\mathrm{Nt})$ is the thermophoresis parameter.

Since the flow is a steady and using the shifts in Eq. (17) and by introducing non-dimensional quantities [Eq.(18)] into constitutive relations (11)-(14), and conduct some algebraic processes we get the following equations:

$\delta \frac{\partial u}{\partial x}+\frac{\partial v}{\partial y}=0$

$\left.\begin{array}{l}\operatorname{Re}\left[\delta(u+1) \frac{\partial u}{\partial x}+v \frac{\partial u}{\partial y}\right]=-\frac{\partial p}{\partial x} \\ +\delta \frac{\partial}{\partial x}\left(S_{x x}\right)+\frac{\partial}{\partial y}\left(\mathrm{~S}_{x y}\right) \\ -\left[M^{2}+\frac{1}{K} \mu(\theta)\right](u+1)\end{array}\right\}$

$$
\begin{aligned}
& \operatorname{Re}\left[\delta(u+1) \frac{\partial v}{\partial x}+v \frac{\partial v}{\partial y}\right]=-\frac{1}{\delta} \frac{\partial p}{\partial y} \\
& +\delta \frac{\partial}{\partial x}\left(S_{x y}\right)+\frac{\partial}{\partial y}\left(S_{y y}\right) \\
& -\frac{d_{1}^{2}}{K_{0}} \mu(\theta) v
\end{aligned}
$$

$\operatorname{Re} \cdot \operatorname{Pr}\left[d(u+1) \frac{\partial q}{\partial x}+v \frac{\partial q}{\partial y}\right]=$

$\left.\left[\begin{array}{c}d^{2} \frac{\partial^{2} q}{\partial x^{2}} \\ +\frac{\partial^{2} q}{\partial y^{2}}\end{array}\right]+N t \cdot P r\left[\begin{array}{c}d^{2}\left(\frac{\partial q}{\partial x}\right)^{2} \\ +\left(\frac{\partial q}{\partial y}\right)^{2}\end{array}\right]\right\}$

$$
\begin{aligned}
S_{x x} & +\lambda_{1}\left\{\delta\left[\frac{\partial}{\partial t}+\left(\frac{\partial \psi}{\partial y}+1\right) \frac{\partial}{\partial x}-\frac{\partial \psi}{\partial x} \frac{\partial}{\partial y}\right] S_{x x}-2 \delta S_{x x} \frac{\partial^{2} \psi}{\partial x \partial y}-2 S_{x y} \frac{\partial^{2} \psi}{\partial y^{2}}\right\} \\
& +\frac{1}{2}\left(\lambda_{1}-\mu_{1}\right)\left[4 \delta S_{x x} \frac{\partial^{2} \psi}{\partial x \partial y}+2 S_{x y}\left(\frac{\partial^{2} \psi}{\partial y^{2}}-\delta^{2} \frac{\partial^{2} \psi}{\partial x^{2}}\right)\right]=2 \delta \frac{\partial^{2} \psi}{\partial x \partial y} \\
S_{x y} & +\lambda_{1}\left\{\delta\left[\frac{\partial}{\partial t}+\left(\frac{\partial \psi}{\partial y}+1\right) \frac{\partial}{\partial x}-\frac{\partial \psi}{\partial x} \frac{\partial}{\partial y}\right] S_{x y}+\delta^{2} S_{x x} \frac{\partial^{2} \psi}{\partial x^{2}}-S_{y y} \frac{\partial^{2} \psi}{\partial y^{2}}\right\} \\
& +\frac{1}{2}\left(\lambda_{1}-\mu_{1}\right)\left(S_{x x}+S_{y y}\right)\left(\frac{\partial^{2} \psi}{\partial y^{2}}-\delta^{2} \frac{\partial^{2} \psi}{\partial x^{2}}\right)=\frac{\partial^{2} \psi}{\partial y^{2}}-\delta^{2} \frac{\partial^{2} \psi}{\partial x^{2}} \\
S_{y y} & +\lambda_{1}\left\{\delta\left[\frac{\partial}{\partial t}+\left(\frac{\partial \psi}{\partial y}+1\right) \frac{\partial}{\partial x}-\frac{\partial \psi}{\partial x} \frac{\partial}{\partial y}\right] S_{y y}+2 \delta^{2} S_{x y} \frac{\partial^{2} \psi}{\partial x^{2}}+2 \delta S_{y y} \frac{\partial^{2} \psi}{\partial x \partial y}\right\} \\
& +\frac{1}{2}\left(\lambda_{1}-\mu_{1}\right)\left[-4 \delta S_{y y} \frac{\partial^{2} \psi}{\partial x}+2 S_{x y}\left(\frac{\partial^{2} \psi}{\partial y^{2}}-\delta^{2} \frac{\partial^{2} \psi}{\partial x^{2}}\right)\right]=-2 \delta \frac{\partial^{2} \psi}{\partial x} \frac{\partial y}{\partial y}
\end{aligned}
$$


Mohammed .R/ Ahmed .M

The low Reynolds number and long wavelength approximation are widely in the solution of issues concerning the peristaltic flows. The long wavelength approximation is based on a supposition that wavelength of the peris-taltic wave is considerably major as compared with the half width of the channel.

And since the stream functions $(\psi)$ is connected with the velocity components by the relations $u=\frac{\partial \psi}{\partial y}, v=-\frac{\partial \psi}{\partial x}$ and $\delta \square 1$ then:

$$
\begin{aligned}
\frac{\partial p}{\partial x} & =\frac{\partial}{\partial y}\left(s_{x y}\right) \\
& -\left[M^{2}+\frac{1}{K} \mu(\theta)\right]\left(\frac{\partial \psi}{\partial y}+1\right)
\end{aligned}
$$

$\frac{\partial p}{\partial y}=0$

$\frac{\partial^{2} \theta}{\partial y^{2}}+N t \cdot \operatorname{Pr}\left(\frac{\partial \theta}{\partial y}\right)^{2}=0$

With

$$
\begin{aligned}
& S_{x x}=\left(\lambda_{1}+\mu_{1}\right) S_{x y} \frac{\partial^{2} \psi}{\partial y^{2}} \\
& S_{x y}+\frac{1}{2}\left(\lambda_{1}-\mu_{1}\right)\left(S_{x x}+S_{y y}\right) \frac{\partial^{2} \psi}{\partial y^{2}} \\
& -\lambda_{1} S_{y y} \frac{\partial^{2} \psi}{\partial y^{2}}=\frac{\partial^{2} \psi}{\partial y^{2}} \\
& S_{y y}=-\left(\lambda_{1}-\mu_{1}\right) S_{x y} \frac{\partial^{2} \psi}{\partial y^{2}}
\end{aligned}
$$

By simplifying Eqs.(29)-(31), we get

$$
S_{x y}=\frac{\frac{\partial^{2} \psi}{\partial y^{2}}}{1-\xi\left(\frac{\partial^{2} \psi}{\partial y^{2}}\right)^{2}}
$$

Here $\xi=\left(\mu_{1}^{2}-\lambda_{1}^{2}\right)$ is the pseudoplastic fluid parameter.
For the simplicity of analysis, most research on fluid mechanics takes fluid viscosity as a constant quantity.But in many processes, the viscosity is a function of heat, and in the present study, we will take this into account by treating viscosity as an exponential function of temperature. Let us take, $\mu(\theta)=e^{-\alpha \theta}$, where $(\alpha)$ is the Reynolds model viscosity parameter, which is a constant. For ( $\alpha \square 1$ ) neglecting the border which contains the powers of $(\alpha)$ more than two, we write

$\mu(\theta)=1-\alpha \theta \quad$ for $\quad \alpha \square 1$

And by offsetting it in Eq. (26), we get

$$
\begin{aligned}
\frac{\partial p}{\partial x} & =\frac{\partial^{3} \psi}{\partial y^{3}}+3 \xi\left(\frac{\partial^{2} \psi}{\partial y^{2}}\right)^{2} \frac{\partial^{3} \psi}{\partial y^{3}} \\
& -\left(N_{1}^{2}-\frac{\alpha}{K} \theta\right)\left(\frac{\partial \psi}{\partial y}+1\right)
\end{aligned}
$$

Where $N_{1}^{2}=M^{2}+\frac{1}{K}$

The Eq.(27) shows that (p) is not a function of (y) of the non-dimensional axial coordinate $\mathrm{Y}$, from this and also of Eq.(34), we get:

$$
\left.\begin{array}{l}
\frac{\partial^{4} \psi}{\partial y^{4}}+3 \xi\left(\frac{\partial^{2} \psi}{\partial y^{2}}\right)^{2} \frac{\partial^{4} \psi}{\partial y^{4}} \\
+6 \xi \frac{\partial^{2} \psi}{\partial y^{2}}\left(\frac{\partial^{3} \psi}{\partial y^{3}}\right)^{2}-\left(\begin{array}{c}
N_{1}^{2} \\
-\frac{\alpha}{K} \theta
\end{array}\right) \frac{\partial^{2} \psi}{\partial y^{2}} \\
+\frac{\alpha}{K} \frac{\partial \theta}{\partial y}\left(\frac{\partial \psi}{\partial y}+1\right)=0
\end{array}\right\}
$$

Similarly, the Eqs. (15) and (16), by using Eqs. (17), (18), then the boundary conditions for the dimensionless stream function and the temperature in the wave frame are:

$$
\left.\begin{array}{l}
\psi=\frac{F}{2}, \frac{\partial \psi}{\partial y}=-1, \theta=0 \text { at } y=h_{1}, \\
\psi=-\frac{F}{2}, \frac{\partial \psi}{\partial y}=-1, \theta=1 \text { at } y=h_{2}
\end{array}\right\}
$$

where $(\mathrm{F})$ is the dimensionless mean flows in the wave frame.where

$$
\begin{aligned}
F(x, t) & =Q+a \operatorname{Sin}[2 \pi x+\phi] \\
& +b \operatorname{Sin}[2 \pi x]
\end{aligned}
$$


Where $(\mathrm{Q})$ is the dimensionless mean flows in the wave frame. Here

$$
\begin{gathered}
F=\int_{h_{1}(x)}^{h_{2}(x)} \frac{\partial \psi}{\partial y} d y \\
\left.=\psi\left(h_{2}\right)-\psi\left(h_{1}\right), Q \equiv \frac{\tilde{q}}{c d}\right) \\
\tilde{q}=\int_{\tilde{H}_{2}(\tilde{x}, t)} \tilde{u}(\tilde{x}, \tilde{y}) d \tilde{y}
\end{gathered}
$$

\section{Solution technique:}

The Eqs. (28) and (35) are cannot find an exact solution to it. And in order to solve this problem, we resort to perturbation method where is applied to find series solving for the small parameters $\left(\mathrm{S}_{\mathrm{xy}}, \psi\right.$, F, p) about fluid parameter $(\xi)$ and $(\theta)$ about Prandtl number (Pr). as shown in the following equations.

$$
\left.\begin{array}{l}
\psi=\psi_{0}+\xi \psi_{1}+\xi^{2} \psi_{2}+\ldots, \\
p=p_{0}+\xi p_{1}+\xi^{2} p_{2}+\ldots, \\
F=F_{0}+\xi F_{1}+\xi^{2} F_{2}+\ldots, \\
\theta=\theta_{0}+\operatorname{Pr} . \theta_{1}+\operatorname{Pr}^{2} . \theta_{2}+\ldots
\end{array}\right\}
$$

furthermore, the series solution is used only up to first order.

\section{Perturbed Systems:}

To find the parameters values we say

(I) The solution (by perturbation technique) for the temperature in Eq.(28) which satisfies the boundary conditions (40), becomes:

(i) Zero order: $\frac{\partial^{2} \theta_{0}}{\partial y^{2}}=0$

(ii) First order: $\frac{\partial^{2} \theta_{1}}{\partial y^{2}}+N t\left(\frac{\partial \theta_{0}}{\partial y}\right)^{2}=0$

With the dimensionless boundary conditions

$$
\begin{aligned}
& \theta_{0}=0, \theta_{1}=0 \text { at } y=h_{1} \text { where } \\
& h_{1}=-1-m(x+t)-a \operatorname{Sin}[2 \pi x+\phi] \\
& \theta_{0}=1, \theta_{1}=0 \text { at } y=h_{2} \quad \text { where } \\
& h_{2}=d+m(x+t)+b \operatorname{Sin}[2 \pi x]
\end{aligned}
$$

(Eq.41) $\Rightarrow \theta_{0}=C_{1}+y C_{2} \quad$ where

$$
\begin{array}{r}
C_{1}=\frac{h_{1}}{h_{1}-h_{2}}, C_{2}=\frac{1}{h_{1}-h_{2}} \\
(\mathrm{Eq} .42) \Rightarrow \theta_{1}=-\frac{N t . \mathrm{y}^{2}}{2\left(h_{1}-h_{2}\right)^{2}}+C_{3}+y C_{4}
\end{array}
$$

Where

$$
C_{3}=-\frac{h_{1} h_{2} N t}{2\left(h_{1}-h_{2}\right)^{2}}, C_{4}=-\frac{-h_{1} N t-h_{2} N t}{2\left(h_{1}-h_{2}\right)^{2}}
$$

Since $\theta=\theta_{0}+\operatorname{Pr} . \theta_{1} \quad$ (by Eq.(44)) we get:

$$
\left.\begin{array}{l}
\theta=\frac{h_{1}}{h_{1}-h_{2}}-\frac{y}{h_{1}-h_{2}}+ \\
\operatorname{Pr}\left(\begin{array}{l}
-\frac{h_{1} h_{2} N t}{2\left(h_{1}-h_{2}\right)^{2}}-\frac{\left(-h_{1} N t-h_{2} N t\right) y}{2\left(h_{1}-h_{2}\right)^{2}} \\
-\frac{N t y^{2}}{2\left(h_{1}-h_{2}\right)^{2}}
\end{array}\right)
\end{array}\right\}
$$

(II) The solution (by perturbation technique) for the momentum equation (35) which satisfies the boundary conditions (40), we get:

$$
\text { (i) zreo order: } \begin{aligned}
\frac{\partial^{4} \psi_{0}}{\partial y}-\left(N_{1}^{2}-\frac{\alpha}{K} \theta\right) \frac{\partial^{2} \psi_{0}}{\partial y^{2}} \\
+\frac{\alpha}{K} \frac{\partial \theta}{\partial y}\left(\frac{\partial \psi_{0}}{\partial y}+1\right)=0
\end{aligned}
$$

With the dimensionless boundary conditions

$$
\begin{aligned}
& \psi_{0}=-\frac{F_{0}}{2}, \frac{\partial \psi_{0}}{\partial y}=-1 \text { at } y=h_{1} \\
& \left.\psi_{0}=\frac{F_{0}}{2}, \quad \frac{\partial \psi_{0}}{\partial y}=-1 \text { at } y=h_{2}\right\}
\end{aligned}
$$

(ii) First order:

$$
\begin{aligned}
\frac{\partial^{4} \psi_{1}}{\partial y^{4}} & +3\left(\frac{\partial^{2} \psi_{0}}{\partial y^{2}}\right)^{2} \frac{\partial^{4} \psi_{0}}{\partial y^{4}}+6 \frac{\partial^{2} \psi_{0}}{\partial y^{2}}\left(\frac{\partial^{3} \psi_{0}}{\partial y^{3}}\right)^{2} \\
& -\left(N_{1}^{2}-\frac{\alpha}{K} \theta\right) \frac{\partial^{2} \psi_{1}}{\partial y^{2}}+\frac{\alpha}{K} \frac{\partial \theta}{\partial y} \frac{\partial \psi_{1}}{\partial y}=0
\end{aligned}
$$

With the dimensionless boundary conditions 


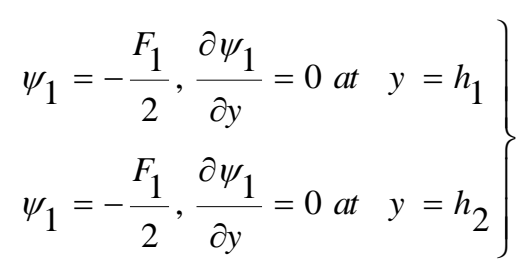

$$
\begin{aligned}
\left(\text { Eq.45 } \Rightarrow \psi_{0}=\right. & a 3+a 4 y+\frac{a 1 \mathrm{e}^{-y N_{1}}+a 2 \mathrm{e}^{y N_{1}}}{N_{1}^{2}}+\alpha(a 7+a 8 y \\
+ & \frac{1}{48\left(h_{1}-h_{2}\right)^{2} K N_{1}^{6}} \mathrm{e}^{-y N_{1}\left(-147\left(a 1+a 2 \mathrm{e}^{2 y N_{1}}\right) N t \cdot P r\right.} \\
+ & 51\left(a 1-a 2 \mathrm{e}^{2 y N_{1}}\right)\left(h_{1}(-2+N t \cdot \operatorname{Pr})+h_{2}(2+N t \cdot \operatorname{Pr})-2 N t \cdot \operatorname{Pr} \cdot y\right) N_{1} \\
& -30\left(a 1+a 2 \mathrm{e}^{2 y N_{1}}\right)\left(-h_{1}+y\right)\left(2 h_{1}-h_{2}(2+N t \cdot \operatorname{Pr})+N t \cdot \operatorname{Pr} \cdot y\right) N_{1}^{2} \\
& -2\left(a 1-a 2 \mathrm{e}^{2 y N_{1}}\right) y\left(-12 h^{2}+y\left(-3 h_{2}(2+N t \cdot \operatorname{Pr})+2 N t \cdot \operatorname{Pr} \cdot y\right)\right. \\
+3 h_{1}\left(2 h_{2}(2+\right. & \left.\left.N t \operatorname{Pr})+(2-N t \cdot \operatorname{Pr}) y)) N_{1}^{3}+48\left(a 5+a 6 \mathrm{e}^{2 y N_{1}}\right)\left(3 h_{1}-3 h_{2}\right)^{2} K N_{1}^{4}\right)\right)
\end{aligned}
$$

$$
\left(\text { Eq.47) } \Rightarrow \psi_{1}=b 3+b 4 y+\frac{1}{8 N_{1}}\left(-2 e^{-y N_{1}}\left(-6 a l^{2} a 2 y-\frac{15 a 1^{2} a 2+4 b 1}{N_{1}}\right)\right.\right.
$$

$$
\begin{aligned}
& \left.+2 e^{y N_{1}}\left(-6 a 1 a 2^{2} y+\frac{15 a 1 a 2^{2}+4 b 2}{N_{1}}\right)-\frac{a 1^{3} e^{-3 y N_{1}}}{N_{1}}-\frac{a 2^{3} e^{3 y N_{1}}}{N_{1}}\right) \\
& \left.-1080 b 1 e^{2 y N_{1}}-8316 a 1 a 2^{2} e^{4 y N_{1}}-1080 b 2 e^{4 y N_{1}}+151 a 2^{3} e^{6 y N_{1}}\right) N t \operatorname{Pr} \\
& -3\left(-72 a l^{2} a 2 e^{2 y N_{1}}\left(12 h_{1}(-2+N t P r)+12 h_{2}(2+N t P r)-37 N t P r y\right)\right. \\
& +72 a l a 2^{2} e^{4 y N} 1\left(12 h_{1}(-2+N t P r)+12 h_{2}(2+N t P r)-37 N t P r y\right) \\
& +35 a l^{3}\left(h_{1}(-2+N t P r)+h_{2}(2+N t P r)-2 N t P r y\right)-7 e^{2 y N_{1}}\left(24 b 1-24 b 2 e^{2 y N_{1}}\right. \\
& \left.\left.+5 a 2^{3} e^{4 y N_{l}}\right)\left(h_{l}(-2+N t P r)+h_{2}(2+N t P r)-2 N t P r y\right)\right) N_{l}+18\left(7 a l^{3}\left(-h_{1}+y\right)\right. \\
& \left(2 h_{1}-h_{2}(2+N t P r)+N t P r y\right)-6 a l^{2} a 2 e^{2 y N_{1}}\left(-68 h_{1}^{2}+7 y\left(-4 h_{2}(2+N t P r)\right.\right. \\
& \left.+5 N t P r y)+h_{1}\left(34 h_{2}(2+N t P r)-28(-2+N t P r) y\right)\right)-6 a l a 2^{2} e^{4 y N_{1}}\left(-68 h_{1}{ }^{2}\right. \\
& \left.+7 y\left(-4 h_{2}(2+N t P r)+5 N t P r y\right)+h_{1}\left(34 h_{2}(2+N t P r)-28(-2+N t P r) y\right)\right) \\
& +\mathrm{e}^{2 y N_{1}}\left(8 b 1\left(10 h_{1}{ }^{2}+3 y\left(h_{2}(2+N t P r)-N t P r y\right)-h_{1}\left(5 h_{2}(2+N t P r)-3(-2+N t P r) y\right)\right)\right. \\
& +\mathrm{e}^{2 y N} 1\left(7 a 2^{3} \mathrm{e}^{2 y N} 1\left(-h_{1}+y\right)\left(2 h_{1}-h_{2}(2+N t \operatorname{Pr})+N t \operatorname{Pr} y\right)+8 b 2\left(10 h_{1}^{2}\right.\right. \\
& \left.\left.\left.\left.+3 y\left(h_{2}(2+N t P r)-N t P r y\right)-h_{1}\left(5 h_{2}(2+N t P r)-3(-2+N t P r) y\right)\right)\right)\right)\right) N_{1}^{2} \\
& +6 y\left(3 a 1^{3}\left(-12 h_{1}{ }^{2}+y\left(-3 h_{2}(2+N t P r)+2 N t P r y\right)+3 h_{1}\left(2 h_{2}(2+N t P r)+(2-N t P r) y\right)\right)\right.
\end{aligned}
$$




$$
\begin{aligned}
& -e^{2 y N_{1}}\left(8 b 1-8 b 2 e^{2 y N_{1}}+3 a 2^{3} e^{4 y N_{1}}\right)\left(-12 h_{1}{ }^{2}+y\left(-3 h_{2}(2+N t \operatorname{Pr})+2 N t \operatorname{Pry}\right)\right. \\
& \left.+3 h_{l}\left(2 h_{2}(2+N t P r)+(2-N t P r) y\right)\right)-6 a l^{2} a 2 e^{2 y N_{1}}\left(-120 h_{l}{ }^{2}+y\left(-39 h_{2}(2+N t P r)\right.\right. \\
& \left.+32 N t P r y)+3 h_{1}\left(20 h_{2}(2+N t P r)-13(-2+N t P r) y\right)\right)+6 a 1 a 2^{2} e^{4 y N_{1}}\left(-120 h_{1}{ }^{2}\right. \\
& \left.+y\left(-39 h_{2}(2+N t P r)+32 N t \operatorname{Pry}\right)+3 h 1\left(20 h_{2}(2+N t P r)-13(-2+N t P r) y\right)\right) N_{1}^{3} \\
& -24\left(2 e ^ { 2 y N _ { 1 } } \left(9 a 2^{2} e^{2 y N_{1}}\left(-10 a 5+a 6 e^{2 y N_{1}}\right)\left(h_{1}-h_{2}\right)^{2} K-2\left(12\left(b 5+b 6 e^{2 y N_{l}}\right)\left(h_{1}-h_{2}\right)^{2} K\right.\right.\right. \\
& \left.\left.-b 4 e^{y N_{1}} y^{2}\left(h 1(6-3 N t P r)-3 h_{2}(2+N t \operatorname{Pr})+2 N t \operatorname{Pry}\right)\right)\right)+3 a 1 a 2 e^{2 y N_{1}}\left(-120 a 5\left(h_{1}-h_{2}\right)^{2} K\right. \\
& +e^{2 y N_{1}}\left(-120 a 6\left(h_{1}-h_{2}\right)^{2} K+a 2 y^{2}\left(-12 h_{1}{ }^{2}+y\left(-3 h_{2}(2+N t \operatorname{Pr})+2 N t \operatorname{Pry}\right)\right.\right. \\
& \left.\left.\left.+3 h_{l}\left(2 h_{2}(2+N t P r)+(2-N t P r) y\right)\right)\right)\right)+3 a l^{2}\left(6 a 5\left(h_{l}-h_{2}\right)^{2} K+e^{2 y N_{1}}\left(-60 a 6\left(h_{l}-h_{2}\right)^{2} K\right.\right. \\
& \left.\left.\left.+a 2 y^{2}\left(-12 h_{1}{ }^{2}+y\left(-3 h_{2}(2+N t P r)+2 N t P r y\right)+3 h_{1}\left(2 h_{2}(2+N t P r)+(2-N t P r) y\right)\right)\right)\right)\right) N_{l}^{4} \\
& \left.\left.-1728 e^{2 y N_{1}}\left(-a 1^{2} a 6+a 2^{2} a 5 e^{2 y N_{1}}-2 a 1 a 2\left(a 5-a b e^{2 y N_{1}}\right)\right)\left(h_{1}-h_{2}\right)^{2} K y N_{1}^{5}\right)\right)
\end{aligned}
$$

And from there it will be

$$
\psi=\psi_{0}+\xi \psi_{1}
$$

Where $\mathrm{a} 1, \mathrm{a} 2, \ldots, \mathrm{a} 8$ and $\mathrm{b} 1, \ldots, \mathrm{b} 6$ are constant . Note also, $u=\frac{\partial \psi}{\partial y}$

(III) The solution for the pressure equation (34) which satisfies the boundary conditions (40) and the Eq. (44):

(i) Zero order :

$$
\begin{aligned}
& \frac{\partial p_{0}}{\partial y}=\frac{\partial^{3} \psi_{0}}{\partial y^{3}} \\
& -\left(N_{1}^{2}-\frac{\alpha}{K} \theta\right)\left(\frac{\partial \psi_{0}}{\partial y}+1\right)
\end{aligned}
$$

(ii) First order:

$$
\begin{aligned}
\frac{\partial p_{1}}{\partial y}= & \frac{\partial^{3} \psi_{1}}{\partial y^{3}}+3\left(\frac{\partial^{2} \psi_{0}}{\partial y^{2}}\right)^{2} \frac{\partial^{3} \psi_{0}}{\partial y^{3}} \\
& -\left(N_{1}^{2}-\frac{\alpha}{K} \theta\right)\left(\frac{\partial \psi_{1}}{\partial y}+1\right)
\end{aligned}
$$

Now by Eqs.(52), (53) and (40) we get:

$$
\left.\begin{array}{c}
\frac{\partial p}{\partial y}=\frac{\partial^{3} \psi_{0}}{\partial y^{3}}-\left(N_{1}^{2}-\frac{\alpha}{K} \theta\right)\left(\frac{\partial \psi_{0}}{\partial y}+1\right) \\
+\xi\left[\begin{array}{c}
\left.\frac{\partial^{3} \psi_{1}}{\partial y^{3}}+3\left(\frac{\partial^{2} \psi_{0}}{\partial y^{2}}\right)^{2} \frac{\partial^{3} \psi_{0}}{\partial y^{3}}\right] \\
-\left(N_{1}^{2}-\frac{\alpha}{K} \theta\right)\left(\frac{\partial \psi_{1}}{\partial y}+1\right)
\end{array}\right\}
\end{array}\right\}
$$

\section{Average pressure rise:}

By The Eq.(54) the pressure rise ( $\Delta p)$ per wavelength and the walls shear stress can be obtained by the formula

$$
\Delta p=\int_{0}^{1} \frac{\partial \mathrm{p}}{\partial \mathrm{x}} d x
$$

\section{Numerical results and discussion :}

In this section, the graphs are a description of values various parameters under the effect of an (MHD) during peristaltic transport for the flow of pseudoplastic nano-fluids in the tapered asymmetric channel through a porous medium with variable viscosity. 
The complicated behavior of the non-Newtonian fluids can be transacted more swiftly with the assist of numerical solutions, where solved numerically using perturbation technique for the nonlinear equations, therefore our numerical approach relies upon the linear equation solvers by Mathematica program to find the results numerically and graphically.

\subsection{The Pressure gradient distribution :}

In this paragraph, describe the effect of different parameters which have an impact on the pressure gradient $(\mathrm{dp} / \mathrm{dx})$ per wavelength. The influence of these parameters is observed for a Figs. (2)-(7), where shown that in the wider part of the channel ($0.7 \leq \mathrm{x} \leq-0.55)$ and $(0 \leq \mathrm{x} \leq 0.2)$, effect these parameters on pressure gradient are very small, which means that the flow can pass easily without imposing a large gradient pressure. Where, in the tight part of the channel $(0.55 \leq \mathrm{x} \leq 0)$, there must be a large pressure gradient in order to keep the same flow of fluid in the channel, especially for the narrowest place of approximately in $(\mathrm{x}=0.25)$ and the values of $(y)$ and $(t)$ are fixed at $y=0.3$ and $t$ $=2$. This is illustrated by the Figs.(2)-(5) the increase of a value of parameters $(\mathrm{M}, \phi, \mathrm{Q}, \xi)$ is leading to the pressure gradient is increasing, but we observe an opposite in Figs.(6)\&(7) where the pressure gradient is decreased when increasing the values of the parameters $(\mathrm{m}, \mathrm{K})$.

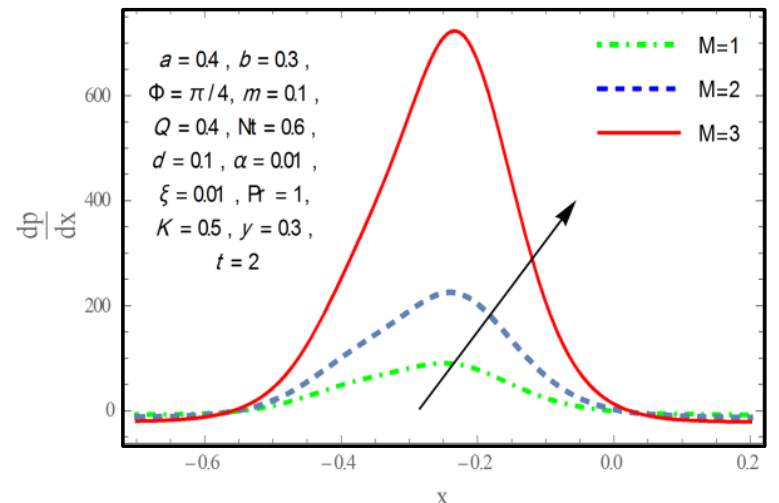

Fig.(2): Variation of $\frac{d p}{d x}$ with increasing of $\mathbf{M}$

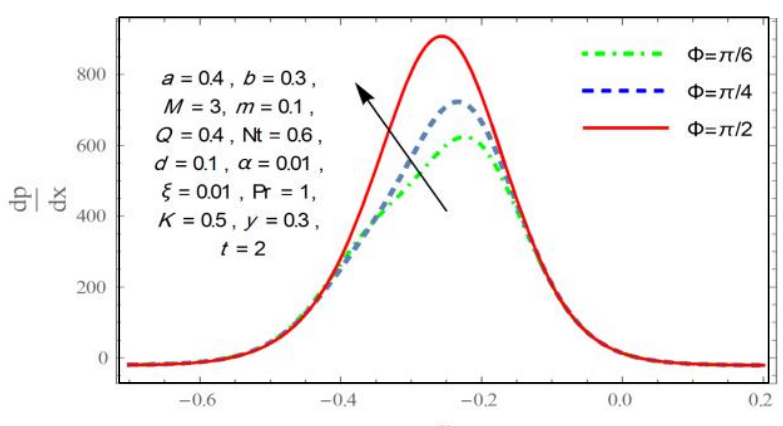

Fig.(3): Variation of $\frac{d p}{d x}$ with increasing of $\phi$

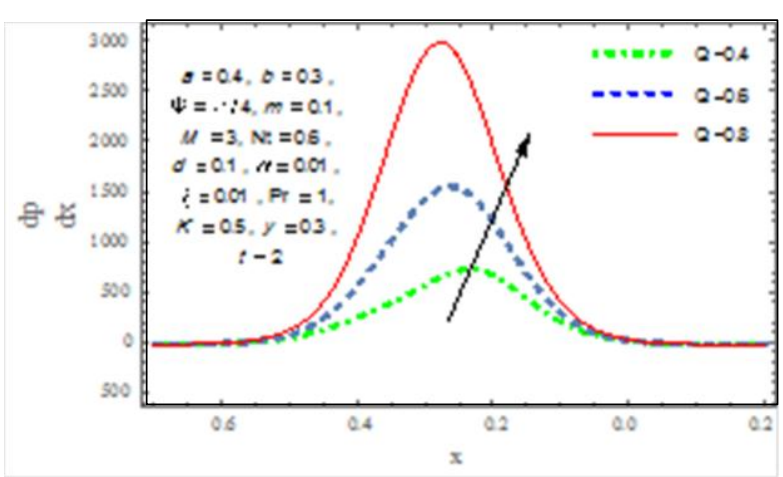

Fig.(4): Variation of $\frac{d p}{d x}$ with increasing of Q

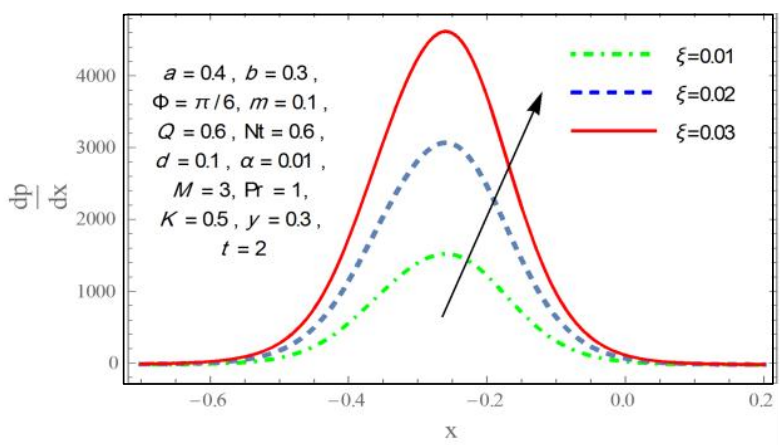

Fig.(5): Variation of $\frac{d p}{d x}$ with increasing of $\xi$

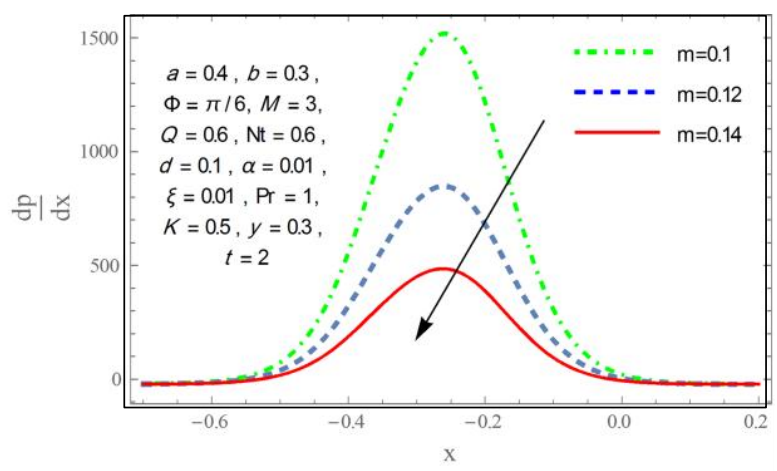

Fig.(6): Variation of $\frac{d p}{d x}$ with increasing of $\mathrm{m}$ 


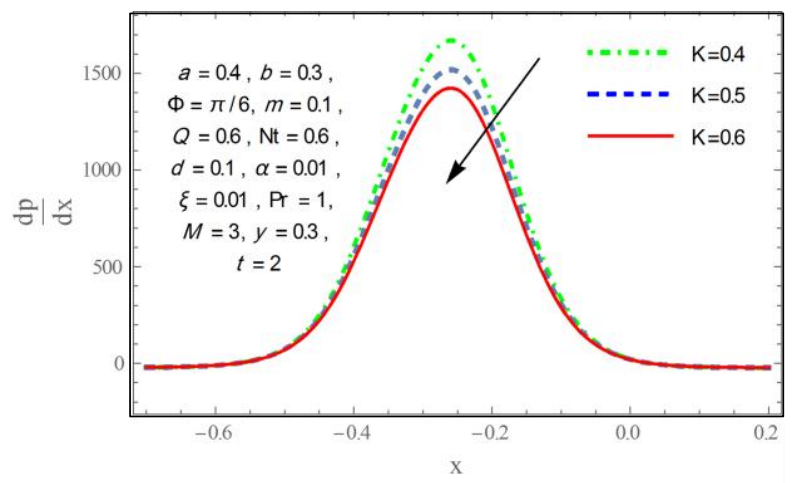

Fig.(7): Variation of $\frac{d p}{d x}$ with increasing of $\mathrm{K}$

\subsection{Pumping Characteristics :}

The Figs.(8)-(13) show the relation between an average pressure rise $(\Delta \mathrm{p})$ and the mean flow rate (Q) with a various physical parameters which are the Hartman number $(\mathrm{M})$, the phase difference $(\phi)$, the pseudoplastic fluid parameter $(\xi)$, the nonuniform parameter $(\mathrm{m})$, the Darcy number $(\mathrm{K})$ and the Prandtl number (Pr). And effect these parameters on the average pressure rise $(\Delta p)$. We observe in Fig.(8) the pumping rate decrease in the co-pumping region

$(\mathrm{Q}>0, \Delta \mathrm{p}<0)$ while the opposite is happening in the retrograde pumping region $(\mathrm{Q}<0, \Delta \mathrm{p}>0)$

with an increase of parameter (M). Illustrated by the Fig.(9), the pumping rate increase in the co-

pumping region $(\mathrm{Q}>0, \Delta \mathrm{p}<0)$ and decreasing in the retrograde pumping region $(\mathrm{Q}<0, \Delta \mathrm{p}>0)$ with an increase in (m). Fig.(10) shows the effect of $(\xi)$ on $\Delta \mathrm{p}$, where the pumping rate decrease with an increase in $(\xi)$ in the co-pumping region $\Delta \mathrm{p}<0$. Fig.(11) shows the impact of $(\phi)$ on average pressure rise $(\Delta \mathrm{p})$, in a co-pumping region $(\mathrm{Q}>0, \Delta \mathrm{p}<0)$ is increased up to point $(0.93,-29.73)$ but the situation is reflected after that point, where there is a decrease in the pressure rate with enhancing of $(\phi)$. The Fig. (12), demonstrate the effect of $(\mathrm{K})$ on $(\Delta \mathrm{p})$. It is noted that $(\Delta \mathrm{p})$ is increasing in the co-pumping region $(\mathrm{Q}>0, \Delta \mathrm{p}<0)$ with values enhancing $(\mathrm{K})$. Finally, the effect of the parameter (Pr) is very simple is negligible on $\Delta \mathrm{p}$, this is illustrated by Fig. (13).
Mohammed .R/ Ahmed .M

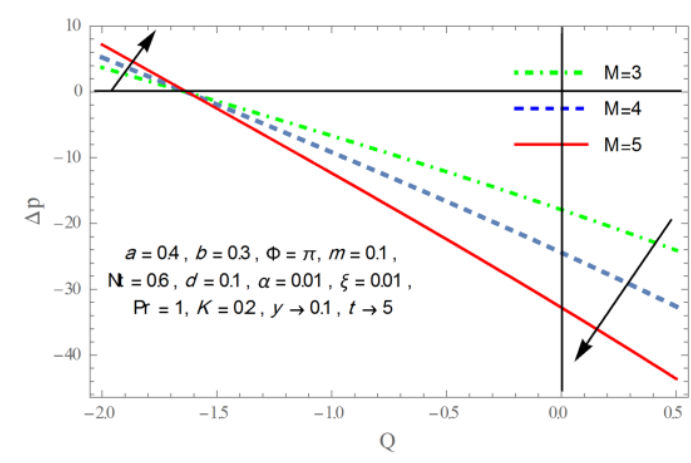

Fig.(8): Variation of $\Delta p$ with increasing of $\mathrm{M}$

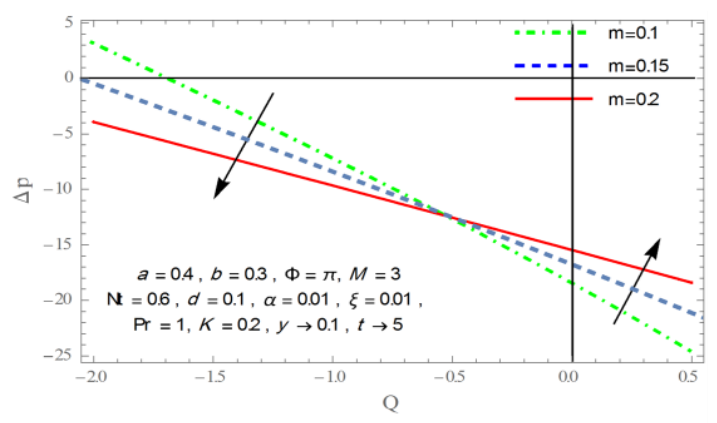

Fig.(9): Variation of $\Delta p$ with increasing of $\mathrm{m}$

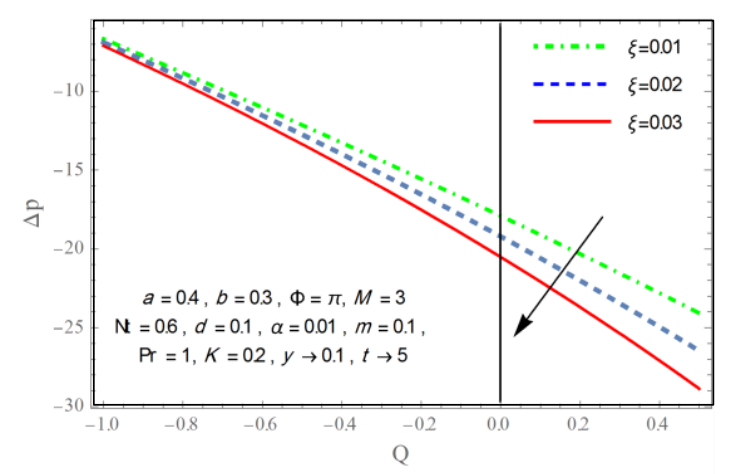

Fig.(10): Variation of $\Delta p$ with increasing of $\xi$

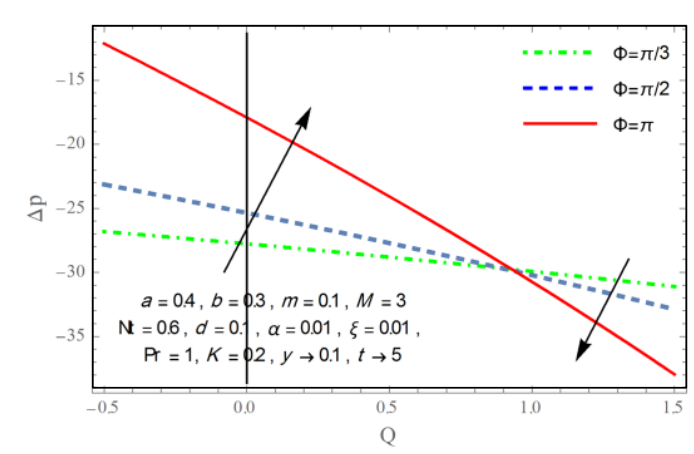

Fig.(11): Variation of $\Delta p$ with increasing of $\phi$ 


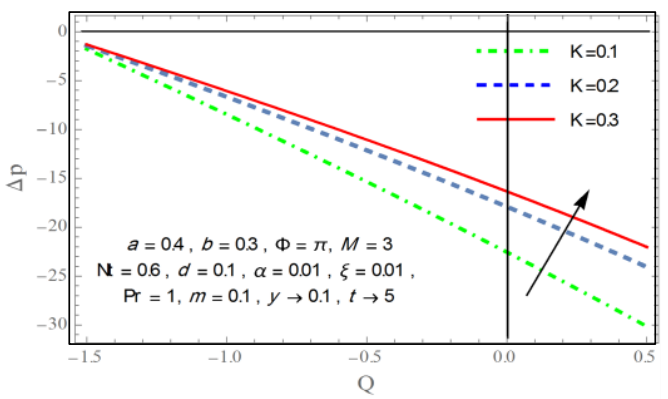

Fig.(12): Variation of $\Delta p$ with increasing of $\mathrm{K}$

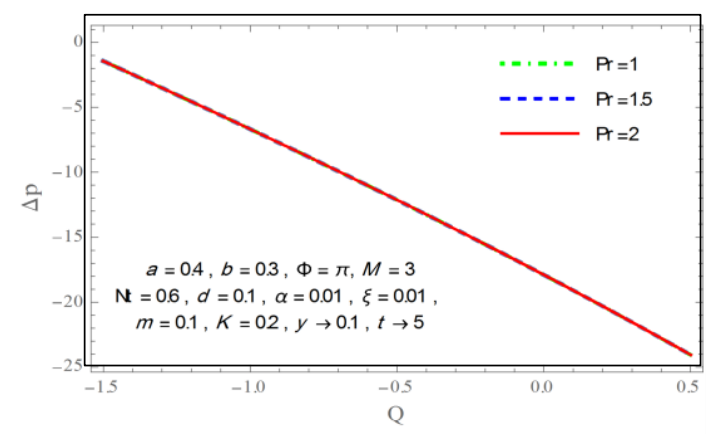

Fig.(13): Variation of $\Delta p$ with increasing of $\operatorname{Pr}$

\subsection{Velocity Profile :}

The Fig.(14)-(17) illustrate the velocity profile (u) versus y-axis, and to see the effects of a change in the values for different parameters at the fixed values of $\mathrm{x}=0.3$ and $\mathrm{t}=2$. The behavior of velocity profile is parabola as seen through figures.We observed from the Fig.(14) that the axial velocity (u) increases with an increase in the Hartmann number (M) at the core part of the channel, but it decreasing for near to walls where this result is expected because the fact that an effect of magnetic field generates a Lorentz force which is a resistant force.This force tends to oppose the fluid movement causing the flux to decelerate. In Fig.(15) we observe the opposite. The velocity decreases in the center and increases near the walls by increasing the values of the thermophoresis parameter $(\mathrm{Nt})$. The Figs.(16)\&(17) shows the effect of the Darcy number $(\mathrm{K})$, the mean flow rate $(\mathrm{Q})$ on the velocity profile $(\mathrm{u})$, where the velocity decreases with an increase values for these parameters, in the center of the channel .

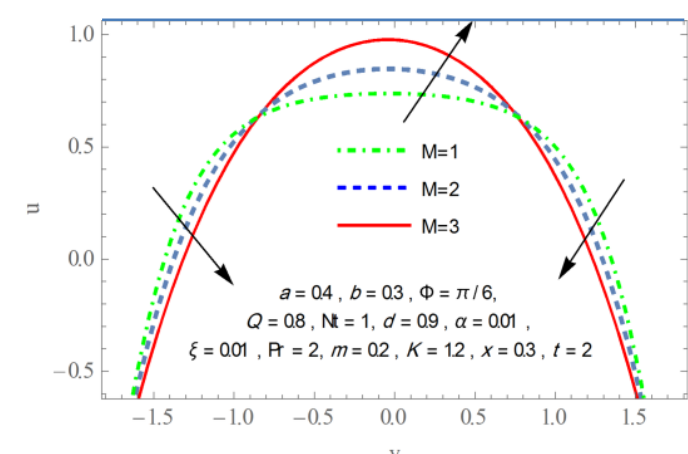

Fig.(14): Variation of (u) with increasing of $\mathrm{M}$

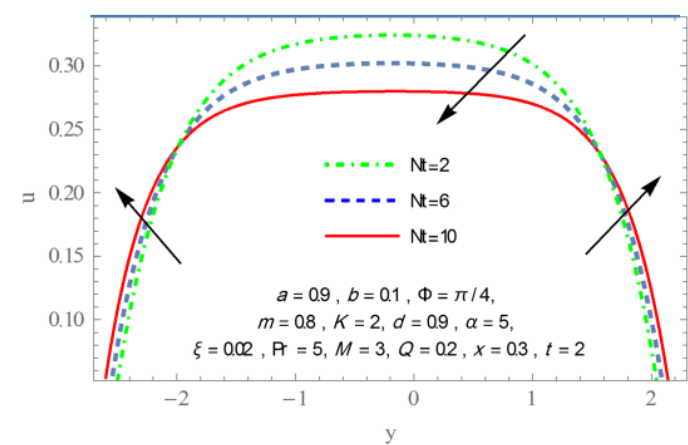

Fig.(15): Variation of $(\mathrm{u})$ with increasing of $\mathrm{Nt}$

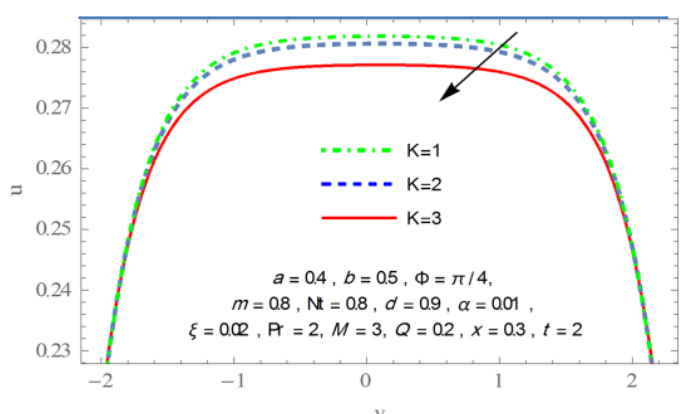

Fig.(16): Variation of $(\mathrm{u})$ with increasing of $\mathrm{K}$

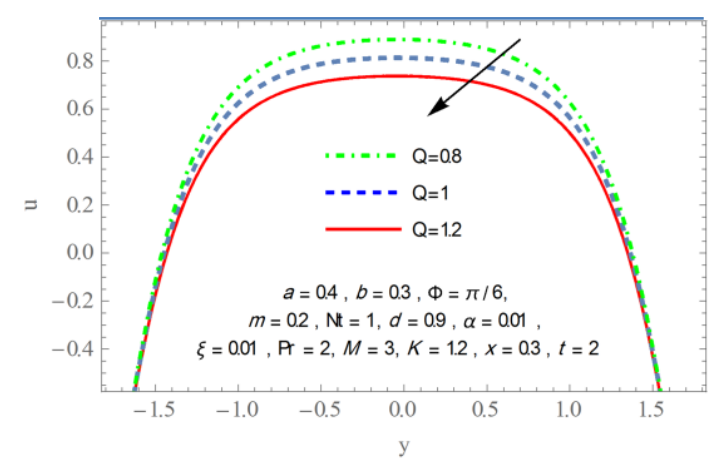

Fig.(17): Variation of $(\mathrm{u})$ with increasing of $\mathrm{Q}$ 


\subsection{Temperature distribution:}

The Figs.(18)-(21) shows the variation in the distribution of temperature between the center of the channel and the layers near the walls, during the peristaltic motion of the fluid for the fixed values of $\mathrm{x}=1$ and $\mathrm{t}=0.5$.

The Figs.(18)\&(19) illustrate the effect a phase difference $(\phi)$ and the non-uniform parameter $(\mathrm{m})$ on the temperature of fluid $(\theta)$,

where $(\theta)$ is increasing near to walls with enhances to $(\phi \& \mathrm{~m})$, but the temperature is almost not affected by with height values of those parameters in center of the channel. While is the opposite with parameters $(\mathrm{Nt})$ and $(\mathrm{Pr})$ where the temperature of the fluid is decreasing near to walls of the channel and the effect of these parameters is gradually fading in the middle of the channel [see Figs.(20)\&(21)].

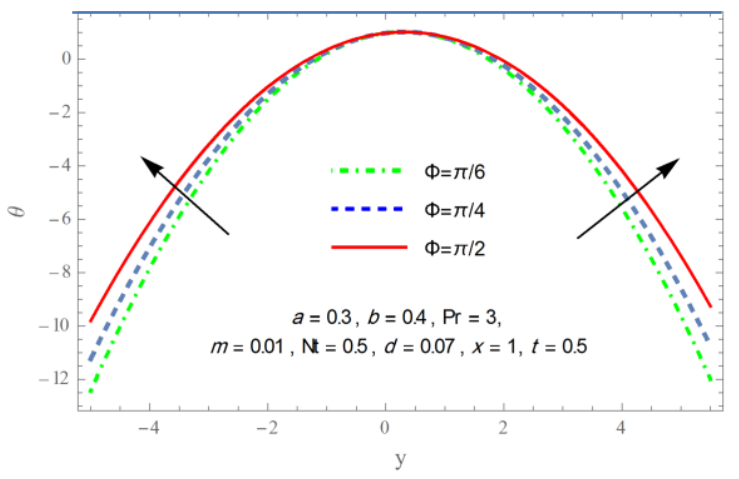

Fig.(18): Variation of $(\theta)$ with increasing of $\phi$

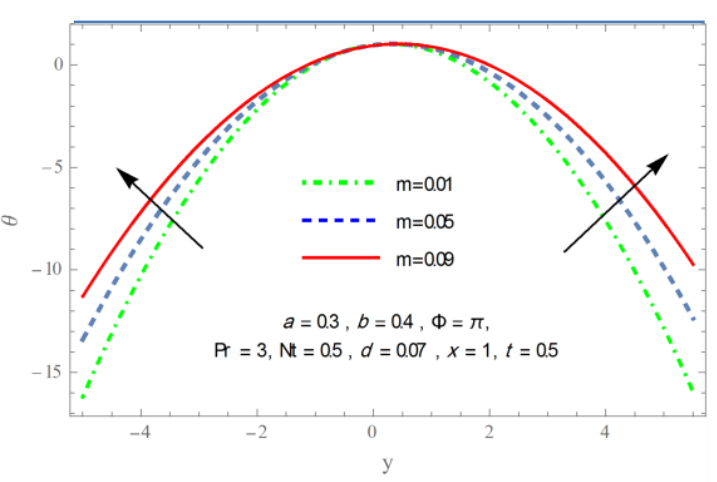

Fig.(19): Variation of $(\theta)$ with increasing of $\mathrm{m}$

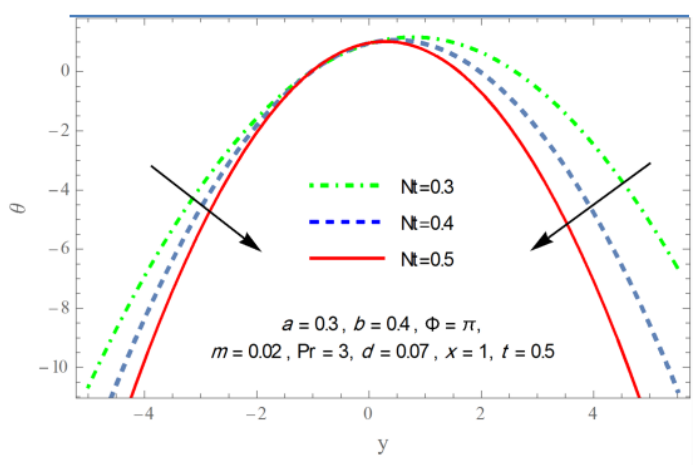

Fig.(20): Variation of $(\theta)$ with increasing of $\mathrm{Nt}$

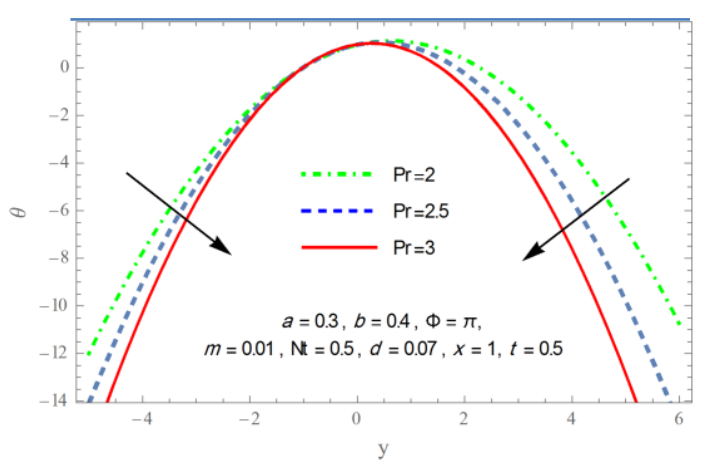

Fig.(21): Variation of $(\theta)$ with increasing of $\operatorname{Pr}$ 

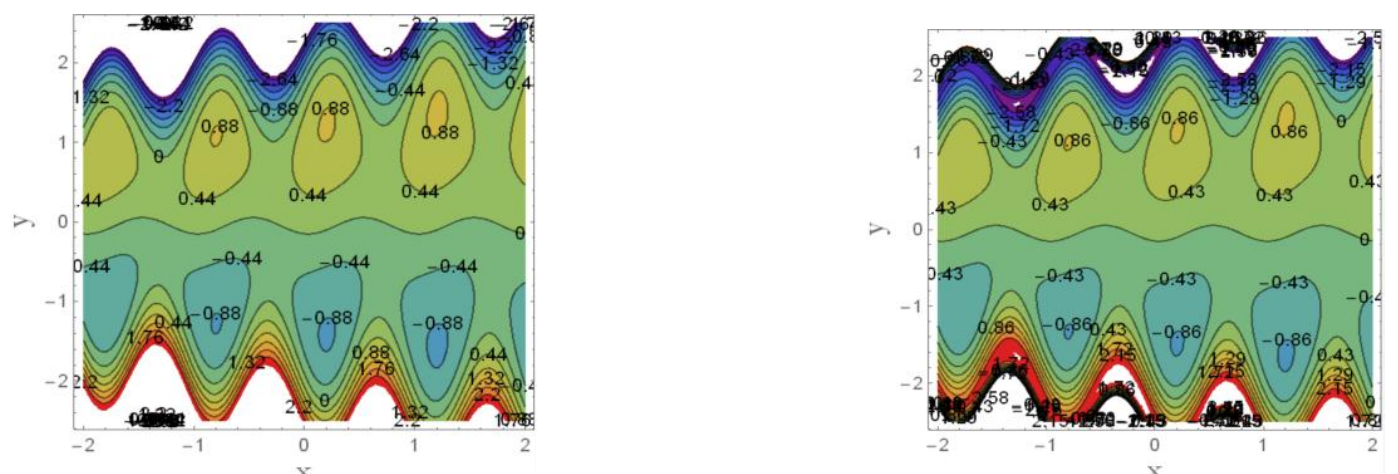

Fig.(22): Streamlines for $a=0.4, b=0.3, \phi=\pi / 6, m=0.2, Q=0.8, N t=1.1, d=0.9, \alpha=0.01$, $\xi=0.002, \operatorname{Pr}=1, K=1.2, t=2$ and for different $\mathbf{M}:$ (a) $\mathbf{M = 1}$, (b) $\mathbf{M = 2}$.
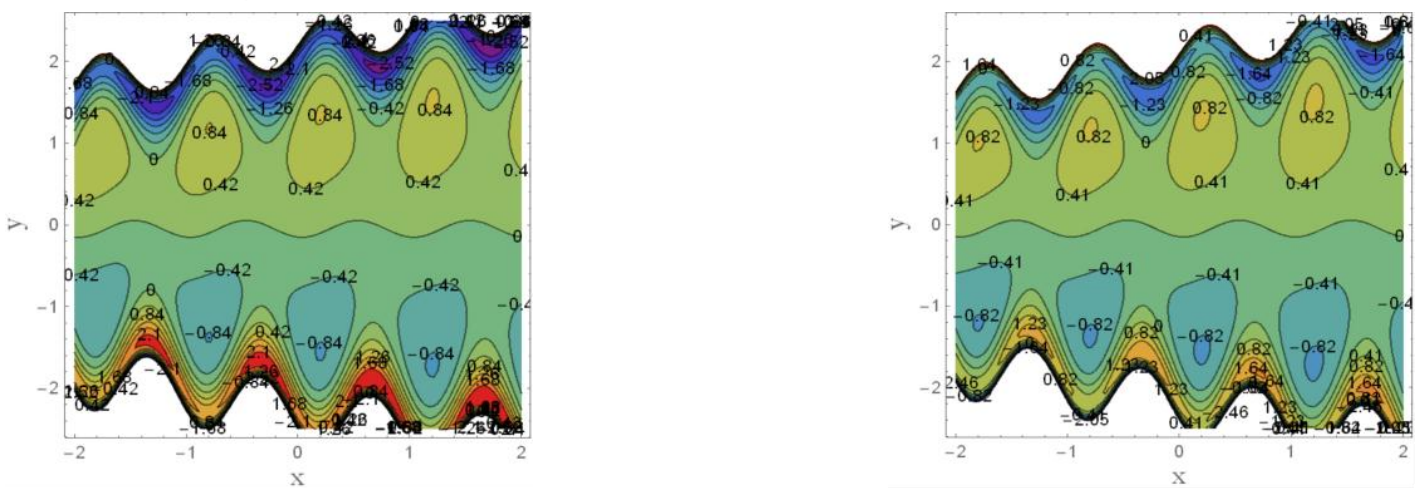

Fig.(23): Streamlines for $a=0.4, b=0.3, \phi=\pi / 6, m=0.2, Q=0.8, N t=1.1, d=0.9, \alpha=0.01$,
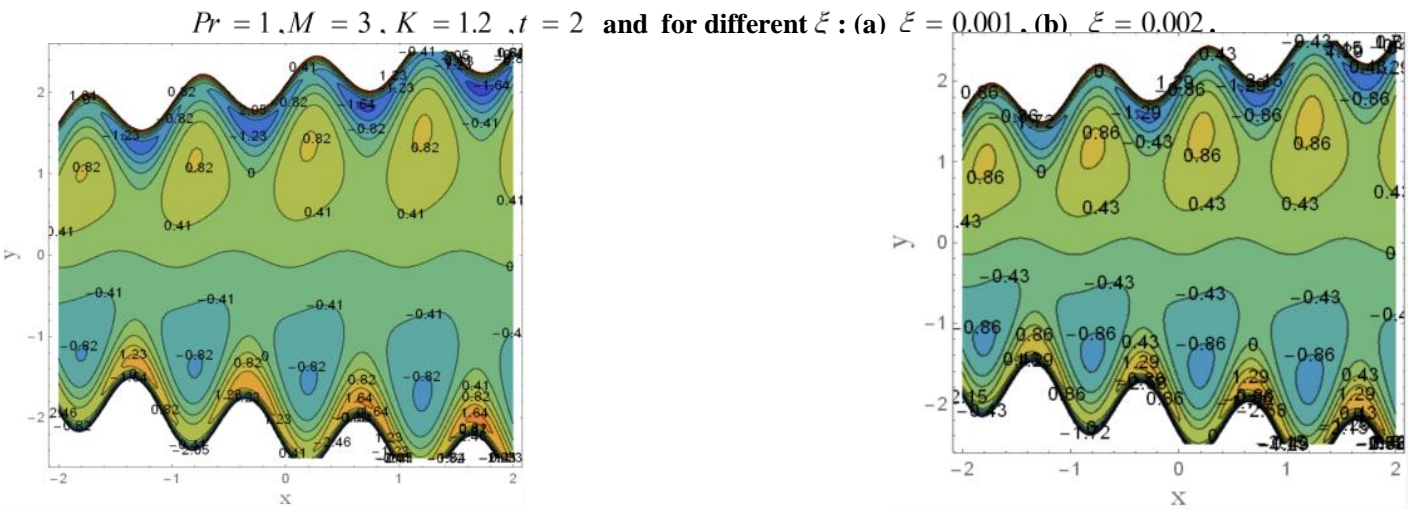

Fig.(24): Streamlines for $a=0.4, b=0.3, \phi=\pi / 6, m=0.2, N t=1.1, d=0.9, \alpha=0.01, \xi=0.002$, $\operatorname{Pr}=1, M=3, K=1.2, t=2$ and for different $\mathbf{Q}:$ (a) $\mathbf{Q = 0 . 8 , ~ ( b ) ~} \mathbf{Q = 1}$.
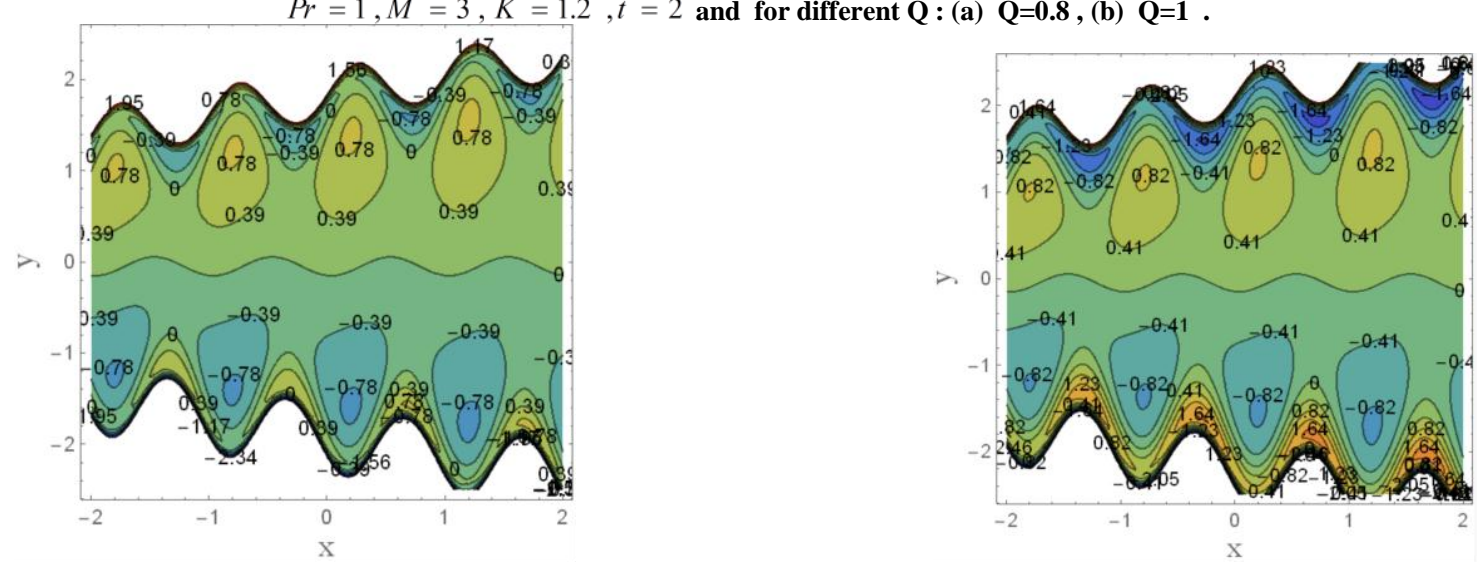

Fig.(25): Streamlines for $a=0.4, b=0.3, \phi=\pi / 6, m=0.2, Q=0.8, N t=1.1, d=0.9, \alpha=0.01$, $\xi=0.002, M=3, \operatorname{Pr}=1, t=2$ and for different $\mathrm{K}:$ (a) $\mathrm{K}=0.1$, (b) $\mathrm{K}=2.2$. 


\section{Conclusions:}

In this paper, we succeeded in presenting a mathematical model to study the effect of no-slip conditions with variable viscosity on peristaltic transport of a non-Newtonian pseudoplastic fluid inside an asymmetric channel. A regular perturbation method is employed to obtain the expression for the pressure gradient and pressure rise over a wavelength, velocity, temperature distribution, the heat transfer coefficient, the Nusselt number and the stream function.

We have discussed the effect peristaltic flow and the rheological parameters of the fluid.

- The increases of a value of parameters $(\mathrm{M}, \phi, \mathrm{Q}, \xi)$ is leading to the pressure gradient is increased and decreased when increasing the values of the parameters $(\mathrm{m}, \alpha, \mathrm{K})$.

- The pumping rate $(\Delta \mathrm{p})$ is decre-asing with an increase in $(\mathrm{M}, \xi)$, But the opposite happens with the parameter $(\mathrm{K})$ in the co-pumping region.

- The profiles of axial velocity (u) take parabolic shape for it is curves.

- The axial velocity (u) increases with the increase in $(\mathrm{M})$ at the core part of the channel but it decreases for near to walls, and the opposite happens with the parameters $(\mathrm{Nt})$.

- The axial velocity (u) decreases with an increase in (K, Q).

- The temperature increases near to walls and almost not affected in center of the channel with enhances to $(\phi, \mathrm{m})$ and the opposite with parameters $(\mathrm{Nt}, \mathrm{Pr})$.

- The size of the trapping bolus increases with increasing of the parameters $(\mathrm{Q}, \mathrm{K})$, while it has decrease with increases of parameters $(\mathrm{M}, \xi)$.

\section{References}

[1] T.W. Latham, Fluid Motions in a Perista-ltic Pump (M.S. Thesis) MIT, Cambrid-ge, MA, 1966.

[2] J.C. Misra,S.K. Pandey, Peristaltic trans-port of physiological fluids, in: J.C. Misra (Ed.), Biomathematics: Modelling and Simulation, World Scientific, London, USA, Singapore, 2006, pp. 167-193 (Chapter 7).

[3] S. Rashidi, M. Dehghan, R. Ellahi,M. Riaz, M.T.Jamal-Abad,Study of stream wise transverse magnetic fluid flow with heat transfer around an obstacle embedded in a porous medium, J. Magn. Magn. Mater. 378 (2015) 128-137.

[4] A. Zeeshan, R. Ellahi, M. Hassan, Magnetohydrodynamic flow of water/ethylene glycol based nano fluids with natural convection through a porous medium, Eur. Phys. J.Plus 129 (12) (2014) 1-10.

[5] M. Sheikholeslami, R. Ellahi, H.R. Ashorynejad, G. Domairry ,T. Hayat, Effects of heat transfer in flow of nano fluids over a permeable stretching wall in a porous medium,J. Comput. Theor. Nanosci. 11 (2) (2014) 486-496.

[6] R. Ellahi, X.Wang, M. Hameed, Effects of heat transfer and nonlinear slip on the steady flow of Couette fluid by means of Chebyshev spectral method, Z.Naturf-orsch., A 69 (1-2) (2014)1-^.

[7] R. Ellahi, E.Shivanian, S.Abbasbandy,T. Hayat, Analysis of some magnetohydrodynamic flows of third order fluid saturating porous space, J. Porous Med. 18 (2) (2015) $89-98$.

[8] K.S. Mekheimer, A.M. Salem, A.Z. Zaher, Peristaltically induced MHD slip flow in a porous medium due to a surface acoustic wavy wall, J. Egypt. Math. Soc. 22 (2014) 143-151.

[9] T. Hayat, S. Noreen, M. Qasim, Influence of heat and mass transfer on peristaltic transport of Phan-Thien-Tanner fluid, $\mathrm{Z}$. Naturforsch., A 68 (2013) 751-758. 
[10] O.U. Mehmood, N. Mustapha, S. Shafie, Heat transfer on peristaltic flow of fourth grade fluid in inclined asymmetric channel with partial slip, Appl. Math. Mech. 33 (2012) $1313-1328$.

[11] S.A. Shehzad, F.M.Abbasi, T.Hayat, F. Alsaadi, G. Mousa, Peristalsis in a curved channel with slip condition and radial magnetic field, Int. J. Heat Mass Transf. 91 (2015) 562-569.

[12] Q. Hussain, S. Asghar, T.Hayat, A. Alsaedi, Heat transfer analysis in peristaltic flow of MHD Jeffrey fluid with variable thermal conductivity, Appl. Math. Mech. 36 (2015) $499-516$.

[13] T. Hayat, S. Noreen, M.S. Alhothuali, S. Asghar, A.Alhomaidan, Peristaltic flow under the effects of an induced magnetic field and heat and mass transfer, Int. J. Heat Mass Transf. 55 (2012) 443 - 452.

[14] D.Tripathi, S.K. Pandey, O.A. Bég, Mathematical modelling of heat transfer effects on swallowing dynamics of viscoelastic food bolus through the human oesophagus, Int. J. Therm. Sci. 70 (2013) 41- 53.

[15] T. Hayat, A. Tanveer, F. Alsaadi, G. Mousa, Impact of radial magnetic field on peristalsis in curved channel with convective boundary conditions, J. Magn. Magn. Mater. 403 (2016) 47-59.

[16] S.U.S. Choi, Enhancing thermal conductivity of fluid with nanoparticles developments and applications of non-Newtonian flow, ASME FED 66 (1995) 99-105.

[17] N. Ali, Q. Hussain, T. Hayat, S. Asghar, Slip effects on the peristaltic transport of MHD fluid with variable viscosity, Phy. Letters, A 372, 1477-1489 (2008).

[18] D.Tripathi, A mathematical model for the move-ment of food bolus of varying viscosities through the oesophagus, Acta Astronautica, 69, 429-439 (2011).

[19] T. Hayat and F. M Abbasi, Variable viscosity effects on the peristaltic motion of a third order fluid, Int. J. Numer. Meth. Fluids, 67, 1500-1515 (2011).
[20] Ebaid, A new numerical solution for the MHD peristaltic flow of a bio-fluid with variable viscosity in a circular cylindrical tube via Adomian decomposition meth-od, Phys. Letters, A 372, 5321-5328 (2008)

[21] S. Nadeem and N. S. Akbar, Effects of heat transfer on the peristaltic transport of MHD Newtonian fluid with variable viscosity: Application of Adomian decomposition method, Commun. Non-linear Sci. and Numer. Simulat.,14, 3844-3855 (2009).

[22] A. Afsar Khan, A. Sohail, S. Rashid, M. Mehdi Rashidi and N. Alam Khan, Effects of Slip Condition, Variable Viscosity and Inclined Magnetic Field on the Peristaltic Motion of a Non-Newtonian Fluid in an Inclined Asymmetric Channel, Journal of Applied Fluid Mechanics,Vol.9, No.3, pp.1381-1393,2016.

[23] T. Hayat, F.M. Abbasi, B. Ahmad, A. Alsaedi, MHD Mixed Convection Peristaltic Flow with Variable Viscosity and Thermal Conductivity, Sains Malay-siana 43(10)(2014): 1583-1590.

[24] F. M. Abbasi, T. Hayat, A. Alsaedi, B. Ahmed, Soret and Dufour Effects on Peristaltic Transport of MHD Fluid with Variable Viscosity, Appl. Math. Inf. Sci.8, No. 1, 211-219 (2014).

[25] J.C. Misra, B. Mallick , A. Sinha , Heat and mass transfer in asymmetric channels during peristaltic transport of an MHD fluid having temperature-dependent properties, AEJ.J. Centre for Healthcare Science, (2016) 711103, India.

[26] A.Sinha, G.C.Shit, N.K. Ranjit, Peristaltic transport of MHD flow and heat transfer in an asymmetric channel: effects of variable viscosity, velocity-slip and temperature jump, Alexandria Eng.J. 54 (2015) 691-704.

[27] T. Hayat, R. Iqbal, A. Tanveer, A. Alsaedi, Influence convective conditions in radiativ peristaltic flow of pseudoplastic nanofluid in a tapered asymmetric channel, J. Magn. Magn. Mater. 408 (2016) 168-176.

[28] T.Hayat, S. Noreen, M.S. Alhothuali, S. Asghar, A. Alhomaidan, Peristaltic flow under of an induced magnetic the effects field and heat and mass transfer, Int. J. Heat 443-452. Mass Transf. 55 (2012). 


\title{
تحليل نقل الحرارة والكتثة في قناة غير متناظرة مدببة اثناء النقل التمعجي لمائع

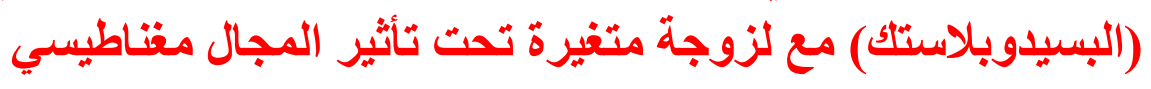

\author{
محمد رزاق سلمان احمد مولود عبد الهادي \\ جامعة بغدادــلية العلوم - قسم الرياضيات
}

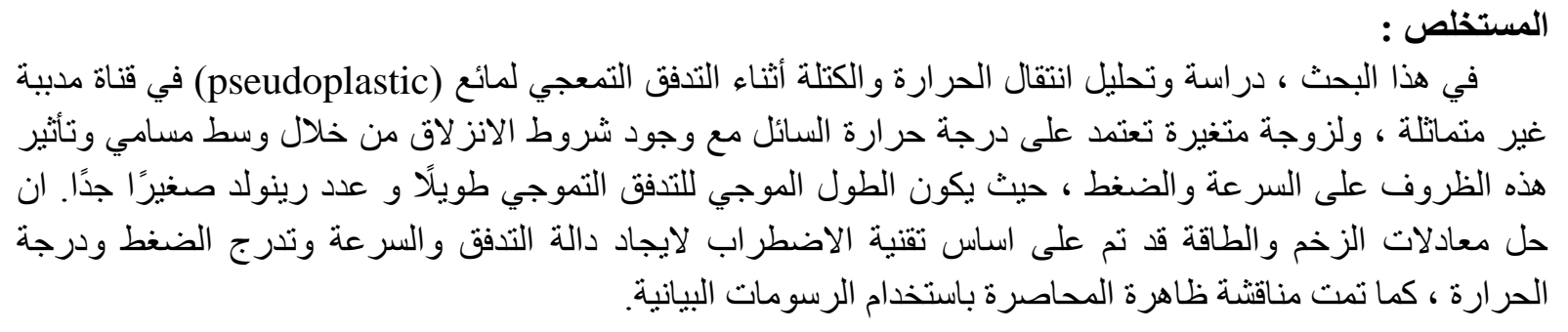

